\title{
Bulk power system availability assessment with multiple wind power plants
}

\author{
Angie C. Cepeda, Mario A. Rios \\ Department of Electrical Engineering and Electronics, School of Engineering, Universidad de los Andes, Colombia
}

\begin{tabular}{l}
\hline \hline Article Info \\
\hline Article history: \\
Received Feb 20, 2020 \\
Revised Jun 14, 2020 \\
Accepted Jun 28, 2020 \\
\hline
\end{tabular}

\section{Keywords:}

Availability assessment Reliability of power systems Wind power plants

\begin{abstract}
The use of renewable non-conventional energy sources, as wind electric power energy and photovoltaic solar energy, has introduced uncertainties in the performance of bulk power systems. The power system availability has been employed as a useful tool for planning power systems; however, traditional methodologies model generation units as a component with two states: in service or out of service. Nevertheless, this model is not useful to model wind power plants for availability assessment of the power system. This paper used a statistical representation to model the uncertainty of power injection of wind power plants based on the central moments: mean value, variance, skewness and kurtosis. In addition, this paper proposed an availability assessment methodology based on application of this statistical model, and based on the $2 \mathrm{~m}+1$ point estimate method the availability assessment is performed. The methodology was tested on the IEEE-RTS assuming the connection of two wind power plants and different correlation among the behavior of these plants.
\end{abstract}

This is an open access article under the CC BY-SA license.

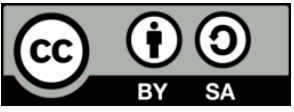

\section{Corresponding Author:}

Mario Alberto Rios,

Department of Electrical Engineering and Electronics,

School of Engineering, Universidad de los Andes,

Carrera 1 No. 18 A - 12, Bogota, Colombia.

Email: mrios@uniandes.edu.co

\section{INTRODUCTION}

The use of renewable energy in bulk power systems, such as wind power plants, have been gaining strength as it brings advantages over traditional sources. Unlike the latter, renewable energies, are clean energy sources which are inexhaustible, abundant and they do not produce greenhouse gases or polluting emissions. However, as these sources dependent on the climatic conditions of the region, the power generated will have a randomly behavior. Therefore, this behavior introduces uncertainties in the performance of bulk power systems [1,2].

In addition, the power system availability has been employed as a useful tool for planning power systems [3]. However, traditional methodologies model generation units as a component with two states: in service or out of service. Nevertheless, this model is not useful to model wind power plants (WPP) for availability assessment of the power system [4], because of a WPP is composed of multiple wind turbines connected by a medium voltage networks to a common connection point (CCP) to the high voltage transmission system [5,6], and the wind is a random variable that makes the power injection of each turbine be a random variable also. As consequence, the total available injection power from a WPP can be represented by a multi-state model where for each power injection can be assigned a probability $[4,7]$; or by the statistical characteristics given by the central moments: mean value, variance, skewness and kurtosis. 
Other authors propose time-varying models of the behavior of WPP, in order to evaluate reliability in operational phase [8]. Thus, [8] proposes an autorregresive moving average model (ARMA), which is not useful for planning purposes. Some authors, by means of Monte Carlo simulation, represent the uncertainty of a WPP only associated to the wind speed without considering the MV network and assume not correlation between several WPPs [9, 10]. Other authors propose WPP probabilistic model for security assessment [11].

On the other hand, it is important to have in mind that within the same power system more than one WPP can be held which could be correlated within a geographical area as they are influenced by the same physical phenomena [12]. Therefore, the availability assessment of a power system most consider correlated wind power plants in the analysis. Furthermore, this assessment is of great importance as it seeks to meet the needs of users as the continuous supply of energy in a secure, reliable way and at the lowest possible cost. For this reason, a reliability analysis is performed in order to determine how well the expected functions of the system are being performed, leading to an improvement in the level of acceptability of the system. Some authors model correlation between winds generators by a copula function to specify multi-variate cumulative distribution functions to be used in a Monte Carlo simulation [13] for the adequacy assessment at generation level.

For the assessment of availability of bulk systems, a model of two states is used to represent the uncertainty of each synchronous generation unit, taking the states of in-service or out-of-service [3, 12]. Nevertheless, wind power plants (WPP) cannot be modelled in two states as shown in [12], as they depend directly on the uncertainty of the wind speed, the operation of the components of the network and the topology of the park [7]. For that reason, different methodologies have been implemented. In [12], a point estimate method (PEM) with $2 \mathrm{~m}$ locations is proposed to simulate uncertainties in load and/or generation for solving the power flow. In addition, as [14] shown, the correlation between two WPP has an impact on the resulting reliability indices by using Monte Carlo's simulation. However, [15] carries out a study that concludes that using a point-estimate method has higher computational efficiency compared to Monte Carlo to solve probabilistic load flow taking into account wind energy sources. Although many PEM schemes have been suggested, among them the $2 \mathrm{~m}+1$ method proposed by Hong [16] allows a high performance when there are a large number of input variables. PEM has been used, recently, to include wind uncertainties in load flow with FACTS devices [17], and analysis of congestion for interconnection of WPP [18].

This paper proposes the application of the method $2 \mathrm{~m}+1$ point-estimate method (PEM) for the availability assessment of a bulk power system considering $\mathrm{m}$ WPPs, where each WPP is a random variable. As the $\mathrm{m}$ WPP have a correlated behavior, a set on independent random variables must be defined by a linear model that correlates the WPP. Using these $m$ independent variables, the method considers 2 concentration points for each random variable ( $m$ variables) around the average value $(+1$, another concentration point); that is the reason of the name of the method [16]. In consequence, in the proposed methodology, the PEM method develops $2 \mathrm{~m}+1$ availability assessments of the bulk power system, where for each evaluation a set of power injection of correlated WPP is computed. In consequence, the method calculates the power generated from the WPP of the system for each $2 \mathrm{~m}+1$ conditions or concentration points and the weighting factor for computing the weighted average of the availability indices. In addition, each WPP is modeled through the four central moments that models the probability distribution function of the injected power by a WPP into the power system and the m number of WPP parks in the electrical system. These central moments can be also computed from statistical data obtained from WPPS.

The independent (section 2.1.) and correlated WPPs central moments (section 2.2) are presented, taking into account [7]. In section 2.3., the method for availability assessment using PEM is shown. Then, in section 3, the proposed PEM-based availability assessment methodology is tested on the IEEE Reliability Test System RTS-24 [19]. Three scenarios were implemented to evaluate the impact of the WPPs in the system; taking a different value of correlation between the injected power by the WPPs. As availability indices, the expected energy non-served (EENS) and the loss of load probability (LOLP) are used. Finally, section 4 presents the conclusions of this paper and further work.

\section{RESEARCH METHOD}

\subsection{Power injection uncertainty in a single wind power plant - statistical model}

Figure 1 shows an example of network configuration of a WPP connected to a point of common coupling (PCC). Typically, a WPP has a number of medium voltage (MV) circuits where the wind turbines are connected through a transformer [20]. All these networks components can be out of service by fails or maintenance, among others. In addition, it is well known that the Wind speed has a random behavior, usually, modeled by a Weibull probability function [21], given by:

$$
f(v)=\left(\frac{b}{a}\right)\left(\frac{v}{a}\right)^{b-1} e^{-\left(\frac{v}{a}\right)^{b}}
$$


Wind speed $(v)$ is an input value that allows the computation of the power generation of each individual wind turbine. However, the wind speed is different for each wind turbine. In (1), $b$ and $a$ are the shape and scale parameters of the Weibull distribution. As [7] shown, a Monte Carlo simulation that takes into account the random state of operation of the network components (in-service or out-of-service), the state of operation of the turbine (in or out of service), and the random characteristics of the wind speed and the wind direction [22]; such as, the power injected at the PCC can be computed for a number of probabilistic realizations. With these simulations a probabilistic distribution function (pdf) can be adjusted, or a discrete pdf can be computed; following these steps:

- Generate the random variables for wind direction and wind speed.

- Randomly, determine the operating status of each turbine (in or out-of-service).

- Calculate the power generation of each wind turbine.

- Generate random variables for each circuit component and determine its operating state (in or out-ofservice).

- Calculate power generated for each circuit and by the wind park by load flow.

- Calculate the 2 central moments mean and variance of the total injected power at the PCC.

- If the convergence criterion of the Monte Carlo simulation is satisfied continue to 8. Otherwise repeat steps $1-6$.

- Obtain the histograms of the density and distribution functions of the power generation of the wind farm

- Perform statistical analysis

Considering the previous steps, in [7] the simulation was carried out for a WPP of $200 \mathrm{MW}, 80$ wind turbines arranged in $10 \mathrm{MV}$ circuits connected like Figure 1 shows, and converged after 1569 realizations. As a result, the power characteristics of the power injection WPP model were obtained. Alternatively, in operating WPP, a historic data can be employed in order to count with a representative sample that allows the statistical modelling of the injected power of the WPP.

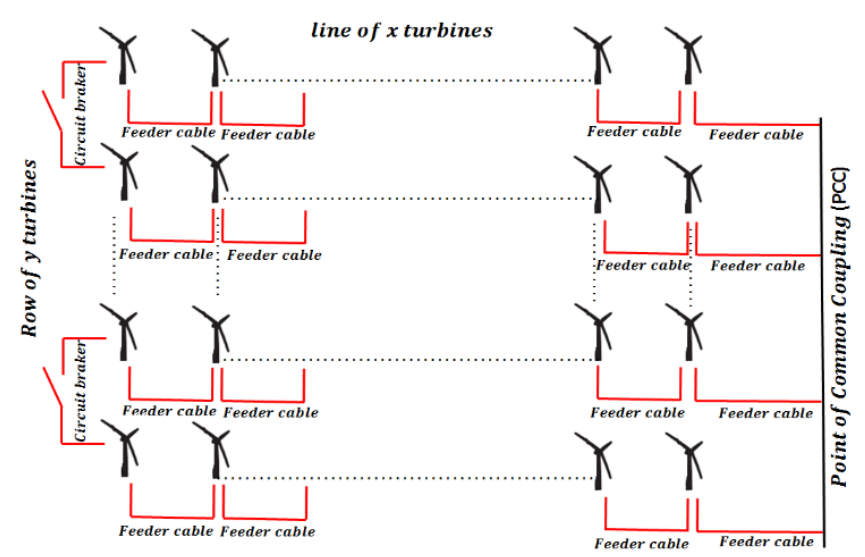

Figure 1. Example of a WPP network

So, from statistical data or from a Monte Carlo simulation; a statistical model of the injected power $\left(\mathrm{P}_{\mathrm{i}}\right)$ by the WPP is fixed computing its four central moments which are: mean or average injected power $\left(\mu_{p}\right)$, its standard deviation $\left(\sigma_{p}\right)$, and its skewness $\left(\lambda_{p, 3}\right)$ and kurtosis $\left(\lambda_{p, 4}\right)$. These four statistics are used by the PEM methodology (as section 2.3 explains), and are defined as follow:

$$
\begin{aligned}
& \mu_{p}=\frac{\sum_{i=1}^{N} P_{i}}{N}=\bar{P}_{l} \\
& \sigma_{p}=\sqrt{\frac{\sum_{i=1}^{N}\left(P_{i}-\bar{P}_{l}\right)^{2}}{N-1}} \\
& \lambda_{p, 3}=\frac{m_{3}}{m_{2}{ }^{3 / 2}} \\
& \lambda_{p, 4}=\frac{m_{4}}{m_{2}{ }^{2}}
\end{aligned}
$$


where, $\mathrm{N}$ is the number of data samples, and

$$
\begin{aligned}
& m_{2}=\frac{\sum_{i=1}^{N}\left(P_{i}-\bar{P}_{l}\right)^{2}}{N} \\
& m_{3}=\frac{\sum_{i=1}^{N}\left(P_{i}-\bar{P}_{l}\right)^{3}}{N} \\
& m_{4}=\frac{\sum_{i=1}^{N}\left(P_{i}-\bar{P}_{l}\right)^{4}}{N}
\end{aligned}
$$

\subsection{Correlated wind power plants uncertainties model}

From section 2.1, each WPP is represented by a statistical model of four statistics computed from the injected power at the PCC: mean or average injected power $\left(\mu_{p}\right)$, its standard deviation $\left(\sigma_{p}\right)$, skewness $\left(\lambda_{p, 3}\right)$, and kurtosis $\left(\lambda_{p, 4}\right)$. Nevertheless, if a power system has $\mathrm{m}$ WPP, the wind behavior of these WWP can be correlated taking into account weather seasons, WWPs locations, among other factors [23]. So, the statistical behavior of a set of WWPs is given not only for their individual statistics $\left(\mu_{p}, \sigma_{p}, \lambda_{p, 3}, \lambda_{p, 4}\right)$ but also by the covariance between the injected power of these WWP. So these covariance of injected power by the WPPs $\left(\left[\mathbf{C}_{\mathbf{p}}\right]\right)$ is given by:

$$
\left[\mathbf{C}_{\mathbf{p}}\right]=\left[\begin{array}{cccc}
\sigma_{p 1}{ }^{2} & \sigma_{p 1 p 2} & \cdots & \sigma_{p 1 p m} \\
\sigma_{p 1 p 2} & \sigma_{p 2}{ }^{2} & \cdots & \sigma_{p 2 p m} \\
\vdots & \vdots & \ddots & \vdots \\
\sigma_{p m p 1} & \sigma_{p m p 2} & \cdots & \sigma_{p m}{ }^{2}
\end{array}\right]
$$

where $\sigma_{p i}$ is the standard deviation of the injected power by the i-th WWP obtained in section 2.1; and $\sigma_{p i p j}$ is the covariance between the power injections of $\mathrm{i}$-th WWP and the $\mathrm{j}$-th park; which is given by:

$$
\sigma_{\text {pipj }}=r_{\text {pipj }} \times \sigma_{p i} \times \sigma_{p j}
$$

where $r_{p \mathrm{i} p \mathrm{j}}$ is the correlation factor between the power injections of $\mathrm{i}$-th WWP and the $\mathrm{j}$-th park [15].

This correlated model is useful to take into account this statistical characteristic that cen be present in a set of WWPs. However, these model must be transformed in a decoupled (non-correlated) set of random variables that allows probabilistic treatment by the Point Estimate Method (proposed in the next section 2.3) and for Monte Carlo simulation, also. Thus, transformation of correlated variables $\boldsymbol{p}=\left[\begin{array}{lll}p_{1} & \cdots & p_{m}\end{array}\right]^{\boldsymbol{T}}$ into a set of non-correlated variables $\mathbf{x}=\left[\begin{array}{lll}x_{1} & \ldots & x_{m}\end{array}\right]^{T}$ is obtained by the following linear transformation:

$$
[x]=[L]^{-1}[p]
$$

Such as the covariance of the non-correlated variables $\mathbf{x}\left(\left[\mathbf{C}_{\mathbf{x}}\right]\right)$ be given by [15]:

$$
\left[C_{x}\right]=[L]^{-1}\left[C_{p}\right]\left[[L]^{-1}\right]^{T}=[I]
$$

where $[\mathbf{L}]$ is computed from the Cholesky decomposition of the covariance matrix $\left[\mathbf{C}_{\mathbf{p}}\right]$, such as $[3,15]$ :

$$
\left[C_{p}\right]=[L][L]^{T}
$$

Now, for the application of PEM (see section 2.3) is necessary to compute the four central moments of each variable $x$. Thus, from the initial variables $\mathbf{p}$, the statistical central moments for the new variables $\mathbf{x}$ are calculated as [15]:

$$
\begin{aligned}
& {\left[\boldsymbol{\mu}_{\boldsymbol{x}}\right]=\left[\boldsymbol{L}^{-\mathbf{1}}\right]\left[\boldsymbol{\mu}_{\boldsymbol{p}}\right]} \\
& \lambda_{x l, 3}=\sum_{r=1}^{m}\left(L_{l r}{ }^{-1}\right)^{3} \lambda_{p, 3} \sigma_{p}{ }^{3} \\
& \lambda_{x l, 4}=\sum_{r=1}^{m}\left(L_{l r}{ }^{-1}\right)^{4} \lambda_{p, 4} \sigma_{p}{ }^{4}
\end{aligned}
$$


where $\left[\boldsymbol{\mu}_{\mathrm{x}}\right]=\left[\begin{array}{lll}\boldsymbol{\mu}_{x 1} & \ldots & \boldsymbol{\mu}_{x m}\end{array}\right]$ is the vector of the average values of the $\mathrm{m}$ variables $x . \lambda_{x l, 3}$ and $\lambda_{x l, 4}$ are the skewness and the kurtosis of the 1-th variable $x$. In addition, as $\left[\mathbf{C}_{\mathbf{x}}\right]=[\mathbf{I}]$, the value of the variance of each $x_{l}$ is 1 ; then $\sigma_{x l}=1$.

\subsection{Availability assessment using point estimate method}

Monte Carlo simulation and state enumeration have been the traditional methodologies used to assess the availability of bulk power systems [3, 24-27]. Some commercial software have reliability assessment tools based on these techniques. These software, traditionally, uses for the assessment of availability/reliability of bulk systems a model of two states to represent the uncertainty of each synchronous generation unit, taking the states of in-service or out-of-service [3]. Nevertheless, as it has shown in sections 2.1 and 2.2, wind power plants (WPP) cannot be modelled in two states, as they depend directly on the uncertainty of the wind speed, the operation of the components of the network and the topology of the park [7], and the correlation between the different WPPs connected to the power system.

The proposal of this paper is to apply the concepts of the Point Estimate Method (PEM) in order to take into account uncertainties model of WPP and correlated behavior of a set of WPP. Although many PEM schemes have been suggested [12-16] and have been used to solve the problem of probabilistic power flow [15, 26], this paper proposes the use of the $2 \mathrm{~m}+1$ PEM proposed by Hong in [16]. The purpose is to compute the common reliability (availability) indices: Loss of Load Probability (LOLP) and Expected Energy Not Supplied (EENS) as are defined at [26, 27], using the state enumeration method.

The PEM-based adequacy assessment here proposed starts computing the set of $2 \mathrm{~m}+1$ concentration points named $\left([\mathbf{p}]_{l, k}, w_{l, k}\right)$, where $[\mathbf{p}]_{l, k}$ is the injected power of the $\mathrm{m}$ WPPs in the power system for the $k$-th concentration $(k=1,2$, and 3$)$ associated to the $l$-th random variable $(l=1,2, \ldots, \mathrm{m})$; and $w_{l, k}$ is the weighting factor computed for this concentration point.

As, the WPPs are correlated, as a general assumption, the $[\mathbf{p}]_{l, k}$ is computed from (11) as:

$$
[\boldsymbol{p}]_{l, k}=[\boldsymbol{L}][\boldsymbol{x}]_{l, k}
$$

It means, that previously it must be computed the concentration points and the weighting factors using the decoupled variables, which are statistically independent. Thus, the vector $[\mathbf{x}]_{l, k}$ is given by:

$$
[x]_{l, k}=\left[\mu_{x 1}, \cdots, x_{l, k}, \cdots, \mu_{x m}\right]
$$

where $\mu_{x 1}$ means that all random variable $x$ i different to the $l$-th random variable takes the first central moment; i.e. the mean value of $x$ i. For each $l$-th random variable are 3 concentration points; i.e. $k=1,2,3$. The $l$-th random variable takes the value

$$
x_{l, k}=\mu_{x l}+\xi_{l, k} \sigma_{x l}
$$

where $\xi_{l, k}$ is the standard location, and $\mu_{x l}, \sigma_{x l}, \lambda_{x l, 3}, \lambda_{x l, 4}$ the central moments of the input random variable $x_{l}$. Thus,

$$
\begin{aligned}
& \xi_{l, k}=\frac{\lambda_{x l, 3}}{2}+(-1)^{3-k} \sqrt{\lambda_{x l, 4}-\frac{3}{4} \lambda_{x l, 3}{ }^{2}} \text { for } k=1,2 \\
& \xi_{l, 3}=0 \quad \text { for } k=3
\end{aligned}
$$

where the skewness $\left(\lambda_{x l, 3}\right)$ and the kurtosis $\left(\lambda_{x l, 4}\right)$ of $x_{l}$ are computed from (15) and (16). Note that for all $l$-th random variable $[\mathbf{x}]_{l, 3}$ is a common vector given by:

$$
[\boldsymbol{x}]_{l, 3}=\left[\mu_{x 1}, \cdots, \mu_{x l}, \cdots, \mu_{x m}\right]
$$

On the other hand, the weighting factor $w_{l, k}$ is computed as:

$$
\begin{aligned}
& w_{l, k}=\frac{(-1)^{3-k}}{\xi_{l, k}\left(\xi_{l, 1}-\xi_{l, 2}\right)} \text { for } k=1,2 \\
& w_{l, 3}=\frac{1}{m}-\frac{1}{\left(\lambda_{x l, 4}-\lambda_{x l, 3}^{2}\right)} \quad \text { for } k=3
\end{aligned}
$$


Once the concentration points $[\mathbf{p}]_{l, k}$ is obtained from (17), an availability assessment of the bulk power system is carried out using state enumeration. In each assessment, the reliability indices EENS and LOLP are computed for every load point of the system and/or for the system. Let $Z_{i}(l, k)$ the reliability index (EENS or LOLP) computed for bus $i$ (i.e. a load bus) using the power injections WPP $[\mathbf{p}]_{l, k}$, the mean value and the standard deviation of the reliability index at bus $i$ considering all the concentration points is obtained as the weighted average, given by:

$$
\begin{aligned}
& \mu_{z_{i}}=\sum_{l=1}^{m} \sum_{k=1}^{3} w_{l, k} Z_{i}(l, k) \\
& E\left[z_{i}^{2}\right]=\sum_{l=1}^{m} \sum_{k=1}^{3} w_{l, k}\left(Z_{i}(l, k)\right)^{2} \\
& \sigma_{z_{i}}=\sqrt{E\left[z_{i}{ }^{2}\right]-\mu_{z_{i}}{ }^{2}}
\end{aligned}
$$

Figure 2 presents the general procedure of availability assessment using PEM. The calculation of concentration points is made applying (17) to (23). The calculation of reliability indexes makes use of (23) to (27). In Figur, $m$ is the number of WPPs in the power system. Note that $k=1,2,3$. When $k=3$, for any $l$, the concentration point is the same, given by (22).

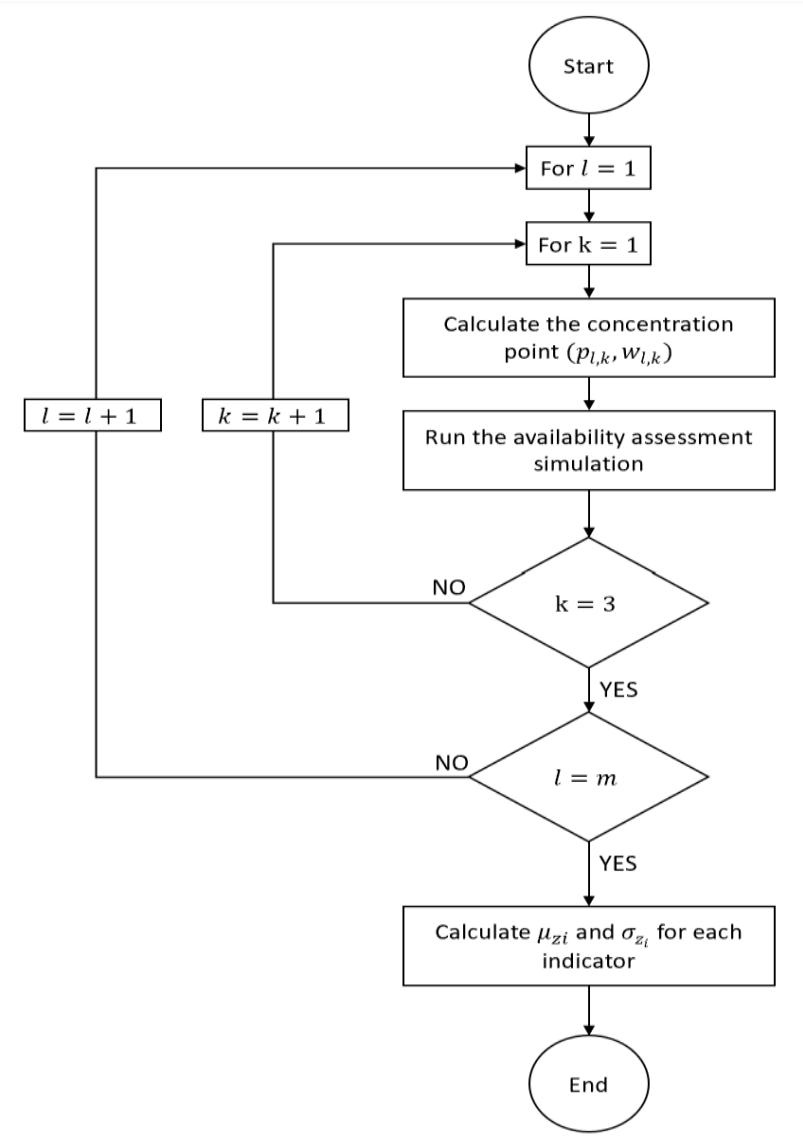

Figure 2. Algorithm of availability assessment using PEM

\section{RESULTS AND ANALYSIS}

\subsection{Study system}

Figure 3 shows the test system, which corresponds to the IEEE Reliability Test System 24 nodes [19], modified. In order to apply the proposed methodology, two WPPs are placed at nodes 7 and 16; replacing the original synchronous generation given at [19]. Each WPP consist of 10 MV circuits with 8 turbines each one, for a total of 80 wind turbines of $2.5 \mathrm{MW}$, based on [5]. In addition, a static var 
compensator device (SVC) is placed in both nodes for voltage regulation of the system. As [7] shows, a Monte Carlo simulation is run to get a statistical characterization of the injected power by the WPP. Table 1 shows this characterization based on a Monte Carlo simulation of 1569 realizations, showing the four central moments $\left(\mu_{p}, \sigma_{p}, \lambda_{p, 3}, \lambda_{p, 4}\right)$.

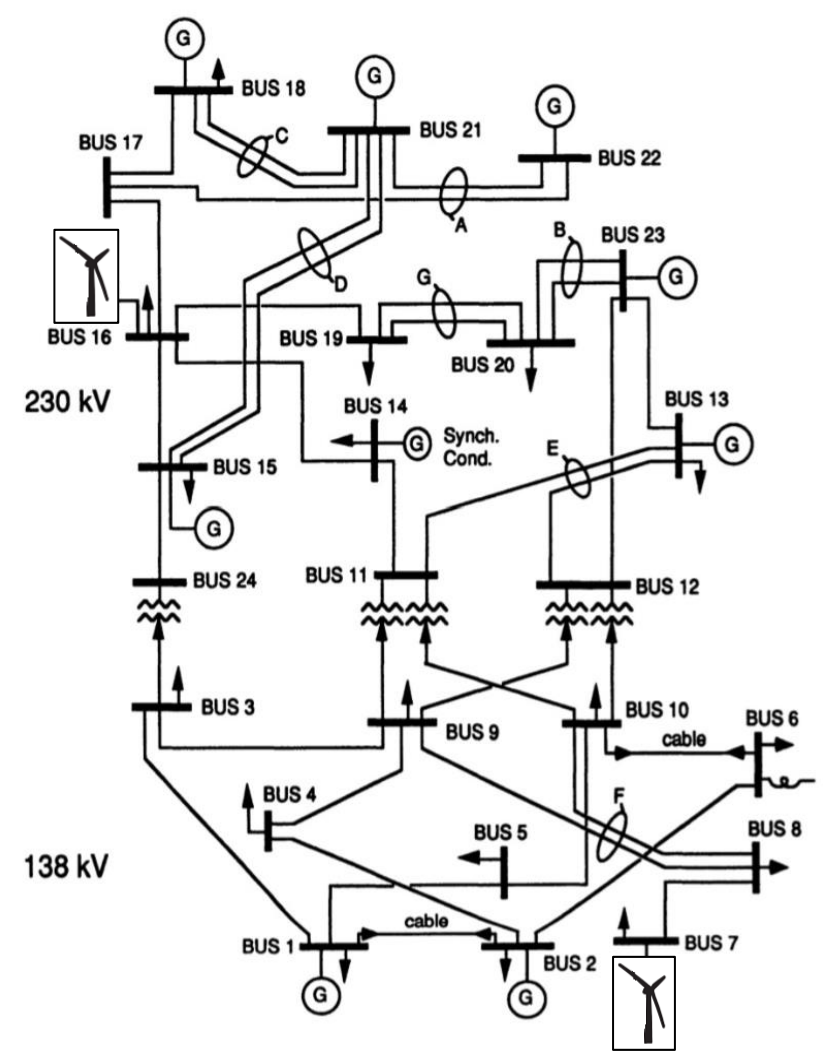

Figure 3. Test system - IEEE reliability test system modified

Table 1. Central moments of the WPP

\begin{tabular}{ll}
\hline Central Moments & Value \\
\hline Mean $\left(\mu_{p}\right)[\mathrm{MW}]$ & 60.36 \\
Standard Deviation $\left(\sigma_{p}\right)[\mathrm{MW}]$ & 60.94 \\
Skewness $\left(\lambda_{p, 3}\right)$ & 0.849 \\
Kurtosis $\left(\lambda_{p, 4}\right)$ & 2.427 \\
\hline
\end{tabular}

\subsection{Study cases}

Three scenarios based on the correlation between the behaviors of power injections of $\mathrm{WPP}_{1}$ (placed at node 16) and $\mathrm{WPP}_{2}$ (placed at node 7) are analyzed. These scenarios are:

- Scenario 1: The injection of both WPPs are assumed as independent random variables; i.e. the correlation $r_{p 1 p 2}=0$.

- Scenario 2: The injection of both WPPs are inversely correlated; assuming a correlation $r_{p 1 p 2}=-0.5$.

- Scenario 3: The injection of both WPPs are directly correlated; assuming a correlation $r_{p 1 p 2}=0.5$.

In this case, there are 2 WPPs, then $m$ takes the value of 2 . In consequence, 5 concentration points $\left(p_{l, k}, w_{l, k}\right)$ are established $(2 \mathrm{~m}+1)$ according to section 2.3. These concentration points are defined at Table 2. It is important to explain the characteristic of the first concentration point:

- Concentration Point 1: this case will be the common concentration point derived from (18). When $\mathrm{k}=3$ for each random variable 1 , both WPP are generating the value of their mean value $\mu_{P 1}$ and $\mu_{P 2}$. In such case $[\mathbf{x}]_{1,3}=[\mathbf{x}]_{2,3}$, then from (17) $[\mathbf{p}]_{1,3}=[\mathbf{p}]_{2,3}$. In addition, the weighting factor of the common concentration point is the sum of the weights $w_{1,3}+w_{2,3}$. 
On the other hand, the other concentration points $[\mathbf{x}]_{l, k}$ are defined based on (18), when $l$ takes the values of 1 and 2 ; and $k=1$ and 2 ( $\mathrm{k}=3$ is the common concentration point). Then, $[\mathbf{x}]_{l, k}$ is transformed to $[\mathrm{p}]_{l, k}$ using (17). Table 2 shows the general definition of the concentration points. The values depend on the scenario under analysis. Table 3 shows for the three scenarios the power generated in MW for each WPP according to the 5 concentration points. Table 4 presents the weighting factor for the concentration points according to Table 2 .

Table 2. Concentration points - test case

\begin{tabular}{cccc}
\hline Concentration Point & Injected Power at WPP & Injected Power at WPP & Weight Factor \\
\hline 1 & $p_{1,3}=\mu_{\mathrm{P} 1}$ & $p_{2,3}=\mu_{\mathrm{P} 2}$ & $w_{1,3}+w_{2,3}$ \\
2 & $\mu_{\mathrm{P} 1}$ & $p_{2,1}$ & $w_{2,1}$ \\
3 & $\mu_{\mathrm{P} 1}$ & $p_{2,2}$ & $w_{2,2}$ \\
4 & $p_{1,1}$ & $\mu_{\mathrm{P} 2}$ & $w_{1,1}$ \\
5 & $p_{1,2}$ & $\mu_{\mathrm{P} 2}$ & $w_{1,2}$ \\
\hline
\end{tabular}

Table 3. Power generated (MW) by WPP - scenarios de analysis

\begin{tabular}{ccccc}
\hline WPP & Concentration Points & \multicolumn{3}{c}{ Correlation Scenarios } \\
& & $r_{p 1 p 2}=0$ & $r_{p 1 p 2}=-0.5$ & $r_{p 1 p 2}=0.5$ \\
\hline Node 16 & 1 & 60.36 & 60.36 & 60.36 \\
& 2 & 60.36 & 60.36 & 60.36 \\
& 3 & 60.36 & 60.36 & 60.36 \\
Node 7 & 4 & 169.93 & 169.93 & 169.93 \\
& 5 & 2.53 & 2.53 & 2.53 \\
& 1 & 60.36 & 60.36 & 60.36 \\
& 2 & 169.93 & 190 & 190.72 \\
& 3 & 2.53 & 8.32 & 0 \\
& 4 & 60.36 & 5.58 & 115.14 \\
& 5 & 60.36 & 89.28 & 31.44 \\
\hline
\end{tabular}

Table 4. Weighting factors by concentration point - scenarios de analysis

\begin{tabular}{cccc}
\hline Concentration Points & \multicolumn{3}{c}{ Correlation Scenarios } \\
& $r_{p 1 p 2}=0$ & $r_{p 1 p 2}=-0.5$ & $r_{p 1 p 2}=0.5$ \\
\hline 1 & -0.172 & 0.001 & 0.109 \\
2 & 0.203 & 0.118 & 0.107 \\
3 & 0.383 & 0.295 & 0.199 \\
4 & 0.203 & 0.203 & 0.202 \\
5 & 0.384 & 0.384 & 0.384 \\
\hline
\end{tabular}

Once the power generation of WPPs are computed for each scenario and its concentration points; the reliability indices following the algorithm of Figure 2 is run. Table 5 presents the system EENS index and Table 6 shows the system LOLP index, computed for each concentration point under each scenario. Applying the weighting factors of Table 4 and (25)-(27) the mean value and the standard deviation of the EENS and the LOLP are computed. The concentration point 1 also represents the case where WPP are assumed as nonrandom variables, i.e. without uncertainty. As the results of the other concentrations, there is an important impact on the uncertainty of the reliability indexes introduced by the uncertainty on the WPPs. On the other hand, the scenario of inverse correlation between $\mathrm{WPP}_{1}$ and $\mathrm{WPP}_{2}$ shows a reduction of the reliability indexes; that is an expected result. By contrast if the WPPs are directly correlated the EENS and LOLP increase.

Table 5. Results of EENS [MWh/yr] - Scenarios de Analysis

\begin{tabular}{cccc}
\hline Concentration Points & \multicolumn{3}{c}{ Correlation Scenarios } \\
& $r_{p 1 p 2}=0$ & $r_{p 1 p 2}=-0.5$ & $r_{p 1 p 2}=0.5$ \\
\hline 1 & 178,449 & 178,449 & 178,449 \\
2 & 46,215 & 32,075 & 31,321 \\
3 & 240,053 & 245,282 & 268,636 \\
4 & 119,911 & 153,439 & 66,687 \\
5 & 229,848 & 148,643 & 250,965 \\
$\mu_{\text {EENS }}[\mathrm{MWh} / \mathrm{yr}]$ & 185,316 & 160,790 & 183,485 \\
$\sigma_{\text {EENS }}[\mathrm{MWh} / \mathrm{yr}]$ & 48,166 & 30,921 & 74,092 \\
\hline
\end{tabular}


Table 6. Results of LOLP [\%] - Scenarios de Analysis

\begin{tabular}{cccc}
\hline Concentration Points & \multicolumn{3}{c}{ Correlation Scenarios } \\
& $r_{p 1 p 2}=0$ & $r_{p 1 p 2}=-0.5$ & $r_{p 1 p 2}=0.5$ \\
\hline 1 & 2.436 & 2.436 & 2.436 \\
2 & 0.799 & 0.667 & 0.677 \\
3 & 2.163 & 2.254 & 2.670 \\
4 & 2.238 & 1.567 & 1.885 \\
5 & 2.471 & 1.943 & 2.937 \\
$\mu_{L O L P}[\%]$ & 2.351 & 2.013 & 2.477 \\
$\sigma_{L O L P}[\%]$ & 0.283 & 0.396 & 0.495 \\
\hline
\end{tabular}

\section{CONCLUSION}

This paper proposed the application of the $2 \mathrm{~m}+1$ point-estimate probabilistic method (PEM) for the availability assessment of a bulk system considering the existence of several correlated WPPs. The proposed methodology, firstly, show how to model the power injection or generation of a wind power plant based on the four statistical central moments: mean value, variance, skewness and kurtosis. Thus, the behavior of WPP can be simulated or analyzed with historical data for obtaining this statistical model. In addition, as the behavior of the power injection of several WPPs can be correlated among them; the proposed methodology makes use of linear transformation in order to decouple them into independent random variables, which are useful for the availability assessment based on PEM.

The 2m+1 PEM methodology used for the availability assessment helps to develop the reliability analysis based on conventional tools (state enumeration or Monte Carlo) based on an appropriate set of powers injected by the WPPs that takes into account theirs individual statistical model (i.e. the four central moments) and the correlated behavior of these power sources. The application of PEM on the reliability of bulk power systems with several wind power parks opens the opportunity to extend the research to the utilization of other non-conventional renewable sources, as the photovoltaic generation (PV farms). Further work is required to make the statistical characterization of PV farms, make statistical studies about the correlation between several WPPs and several PV farms. Thus, further research can be started to measure the impacts of the high level penetration of non-conventional energy sources on reliability performance of the power system; that have an important uncertainty.

\section{REFERENCES}

[1] P. Bie, et al., "Probabilistic dynamic load flow with correlated wind sources," IEEJ Transactions on Electrical and Electronic Engineering, vol. 13, no. 1, pp. 76-83, 2018.

[2] A. S. A. Badawi, et al., "Evaluation of wind power for electrical energy generation in mediterranean cost of Palestine for 14 years," International Journal of Electrical and Computer Engineering (IJECE), vol. 9, no. 6, pp. 4610-4616, 2019.

[3] W. Li, "Risk Assessment of Power Systems," IEEE Press, John Wiley \& Sons., 2005.

[4] A. Moreno, and M. A. Ríos, "Impact of Wind Generation in the Generation Reliability Assessment," Applied Mechanics and Materials (AMM) Journal, vol. 267, pp. 29-32, 2013.

[5] D. van Hertem, O. Gomis-Bellmunt, and J. Liang, "Offshore Wind Power Plants (OWPPS)," Wiley-IEEE Press, pp. 109-138, 2016.

[6] M. de Prada, L. Igualada, C. Corchero, O. Gomis-Belmunt, A. Sumper, "Hybrid AC-DC Offshore Wind Power Plant Topology: Optimal Design," IEEE Transactions on Power Systems, vol. 30, no. 4, pp. 1868-1876, 2015.

[7] R. Vergara, "Probabilistic Model of Wind Power Parks for Reliability Assessment (In Spanish)," Master Degree Thesis in Electrical Engineering, Universidad de Los Andes, Bogota, 2018.

[8] Y. Ding, C. Singh, L. Goel, J. Ostergaard, P. Wang, "Short-Term and Medium-Term Reliability Evaluation for Power Systems with High Penetration of Wind Power," IEEE Transactions on Sustainable Energy, vol. 5, no. 3, pp. 896-906, 2014.

[9] X. Jiang, Z. Zhang, J. Wang, "Studies on the Reliability and Reserve Capacity of Electric Power System with Wind Power Integration," Power Engineering and Automation Conference, 2012.

[10] H. Xiaoqing, Y. Yong, "Wind Power Penetration Limit Calculation based on Power System Reliability," International Conference on Sustainable Power Generation and Supply, pp. 1-4, 2009.

[11] S. A. Peña, M. A. Rios, I. Zubia, "WPP Model in Power System Security Assessment," International Review of Electrical Engineering IREE, vol. 15, no. 2, pp. 155-163, 2020.

[12] C. Su, "Probabilistic Load-Flow Computation Using Point Estimate Method," IEEE Transactions on Power Systems, vol. 20, no. 4, pp. 1843-1851, 2005.

[13] E. Tomasson, L. Söder, "Generation Adequacy Analysis of Multi-Area Power Systems with a High Share of Wind Power," IEEE Transactions on Power Systems, vol. 33, no. 4, pp. 3854-3862, 2017.

[14] W. Wangdee and R. Billinton, "Considering Load-Carrying Capability and Wind Speed Correlation of WECS in Generation Adequacy Assessment,” IEEE Transaction on Energy Conversion, vol. 21, no. 3, pp. 734-741, 2006. 
[15] J. M. Morales, L. Baringo, A. J. Conejo and R. Mínguez, "Probabilistic power Flow with correlated wind sources," IET Generation Transmission and Distribution, vol. 4, no. 5, pp. 641-651, 2010.

[16] H. P. Hong, "An efficient point estimate method for probabilistic analysis," Reliability Engineering \& System Safety, vol. 59, no. 3, pp. 261-267, 1998.

[17] M. El-Azab, W. A. Omran, S. F. Mekhamer, H. E. A. Talaat, "A probabilistic multi-objective approach for FACTS devices allocation with different levels of wind penetration under uncertainties and load correlation," International Journal of Electrical and Computer Engineering (IJECE), vol. 10, no. 4, pp. 3898-3910, 2020.

[18] M.A. Rios, E.G. Guama, "System Congestion Criteria for Interconnection of WPP through VSC-HVDC," IET Generation, Transmission and Distribution, vol. 13, no. 12, pp. 2526-2532, 2019.

[19] Albrecht, Paul F., et al., "IEEE reliability test system," IEEE Transactions on Power Apparatus and Systems, vol. 98, no. 6, pp. 2047-2054, 1979.

[20] Q. Huang, X. Huang, J. Fan, X. Zhang, Y. Wang, "Reliability and economy assessment of offshore wind farms," The Journal of Engineering, vol. 2019, no. 16, pp. 1554-1559, 2019.

[21] A. S. A. Badawi, N. F. Hasbullah, S. Yusoff, A. Hasham, M. Elamassie, "Practical electrical energy production to solve the shortage in electricity in Palestine and pay back period," International Journal of Electrical and Computer Engineering (IJECE), vol. 9, no. 6, pp. 4610-4616, 2019.

[22] G. Erdemir, A. T. Zengin, T. C. Akinci, "Short-term wind speed forecasting system using deep learning for wind turbine applications," International Journal of Electrical and Computer Engineering (IJECE), vol. 10, no. 6, pp. $5779-5784,2020$.

[23] C. L. T. Borges, J. A. S. Dias, "Representation of Wind and Load Correlation in Non-Sequential Monte Carlo Reliability Evaluation," Reliability and Risk Evaluation of Wind Integrated Power Systems, pp. 91-106, 2013.

[24] A. M. Leite da Silva, L. A. F. Manso, A. A. Flavio, M. A. da Rosa, and L. C. Resende, "Composite Reliability Assessment of Power Systems with Large Penetration of Renewable Sources," Reliability and Risk Evaluation of Wind Integrated Power Systems, pp. 107-128, 2013.

[25] R. Billinton, and R. N. Allan, "Reliability Assessment of Large Electric Power Systems," Kluwer Academic Publishers, 1998.

[26] W. Li, "Probabilistic Transmission Planning," IEEE Press John Wiley \& Sons, NJ, USA, 2011.

[27] A. S. Dalabeeh, A. Al-Mofleh, A. R. Alzyoud, H. T. Ayman, "Econocmical and Reliable Expansion Alternative of Composite Power System under Restructuring," International Journal of Electrical and Computer Engineering (IJECE), vol. 8, no. 6, pp. 4790-4799, 2018

\section{BIOGRAPHIES OF AUTHORS}
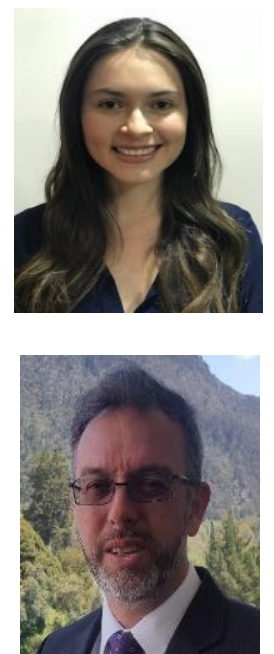

Angie Catalina Cepeda is graduated of electrical engineering from Universidad de los Andes (2020) at Bogotá, Colombia. His interest fields are in electrical power systems and telecommunications systems.e-mail: ac.cepeda@uniandes.edu.co
Mario Alberto Ríos: received a degree in electrical engineering in 1991 and a M.Sc. Degree in electrical engineering in 1992, both from Universidad de los Andes, Bogotá, Colombia. He received a Ph.D. degree in electrical engineering from INPG-LEG, Grenoble, France, in 1998, and a Doctoral degree in engineering from Universidad de los Andes, in 1998. He worked as a consultant engineer in ConCol (now WSP), Bogotá, Colombia, during 12 years. Also, he was a Research Associate at the University of Manchester (formerly, UMIST). Currently, he is Full Professor at the Department of Electrical Engineering, School of Engineering, Universidad de los Andes, Bogotá, and director of the Power and Energy Research Group of this university, 